\title{
FSH Injections and Ultrasonography Determine Presence of Ovarian Components in the Evaluation of Ovotesticular Disorders of Sex Development
}

\author{
Shannon French,, ${ }^{1}$ Luisa Rodriguez,, ${ }^{1}$ Alan Schlesinger, ${ }^{2}$ Laurence McCullough, ${ }^{3}$ \\ Jennifer Dietrich, ${ }^{4}$ John Hicks, ${ }^{5}$ and Lefkothea Karaviti ${ }^{1}$ \\ ${ }^{1}$ Department of Pediatrics, Division of Diabetes and Endocrinology, Gender Medicine Team, \\ Baylor College of Medicine, Houston, TX 77030, USA \\ ${ }^{2}$ Edward B. Singleton Department of Diagnostic Imaging, Texas Children's Hospital, \\ Baylor College of Medicine, TX 77030, USA \\ ${ }^{3}$ Center for Medical Ethics and Health Policy, Gender Medicine Team, Baylor College of Medicine, \\ TX 77030, USA \\ ${ }^{4}$ Department of Obstetrics and Gynecology, Division of Pediatric and Adolescent Gynecology, \\ Baylor College of Medicine, TX 77030, USA \\ ${ }^{5}$ Department of Pathology, Division of Anatomic Pathology, Texas Children's Hospital, \\ Baylor College of Medicine, TX 77030, USA
}

Correspondence should be addressed to Shannon French, slfrench@texaschildrens.org

Received 4 March 2009; Revised 4 August 2009; Accepted 24 October 2009

Recommended by John Achermann

Three infants with ambiguous genitalia and suspected ovotestes were given recombinant FSH to induce ovarian follicular development. The development of follicles in the gonadal tissue suggested the presence of ovarian tissue in two of the three infants. This method may provide a means to better characterize gonadal anatomy in patients affected by disorders of sex development (DSD). Sonographic information poststimulation provided parents with earlier and more specific education and support concerning the possible need for confirmative gonadal biopsy treatment options.

Copyright (C) 2009 Shannon French et al. This is an open access article distributed under the Creative Commons Attribution License, which permits unrestricted use, distribution, and reproduction in any medium, provided the original work is properly cited.

\section{Introduction}

Ovotesticular disorder of sex development (DSD) [1], formerly known as true hermaphroditism, is characterized by the presence of both ovarian and testicular tissue in the gonads of an individual. The traditional approach to establishing a diagnosis of ovotesticular DSD has included history, physical examination, evaluation of chromosomes, sex steroid secretion, imaging of internal anatomy and laparoscopy as the gold standard of establishing the diagnosis. Differentiating the neonatal gonads (testess and ovaries) by sonographic appearance alone is difficult. In the setting of ambiguous genitalia when no clear diagnosis has been established, even with elevated testosterone, ovotestis must be suspected. Performing laparoscopy and biopsy to establish a diagnosis is important for management decisions.
Exogenous FSH has been used for some time in fertility treatments to induce multifollicular development [2]. Pharmacological doses of FSH stimulate the growth of ovarian follicles of various sizes. We report the use of FSH injections in three infants with suspected ovotestes to induce ovarian follicular development, both to determine if the patients would have a responsive ovarian component to their gonads and to enhance the use of sonography in assessing the presence and location of gonads.

\section{Patients and Methods}

2.1. Patients. Three infants (ages 3 and $1 / 2$ weeks, 6 weeks, and 4 months) with ambiguous genitalia, 46XX karyotype, and SRY negative were treated. Descriptions of their external and internal genital structures are shown in Table 1. 
TABLE 1: Description of external and internal genital structures.

\begin{tabular}{lll}
\hline Case & External genitalia & Internal genitalia assessed by US \\
\hline 1 & $\begin{array}{l}1 \mathrm{~cm} \text { phallic structure, tip of urethra identified proximal to } \\
\text { apparent vaginal opening, nonfused labioscrotal structures, } \\
\text { hyperpigmented, nonrugated, no palpable gonads }\end{array}$ & $\begin{array}{l}\text { No uterus: } 0.8 \times 0.5 \mathrm{~cm} \text { gonad visualized in inferior aspect of } \\
\text { the inguinal canal }\end{array}$ \\
$\begin{array}{l}1 \mathrm{~cm} \text { phallic structure, introitus opening with urethra, labio- } \\
\text { scrotal structures partially fused, non-hyperpigmented, right } \\
\text { gonad palpable in inguinal canal }\end{array}$ & $\begin{array}{l}\text { Hypoplastic uterus: } 2.5 \mathrm{~cm} \text { in length with a } 0.2 \mathrm{~cm} \text { fundus, } \\
0.2 \mathrm{~cm} \text { cervix; right inguinal gonad } 0.9 \times 0.6 \times 0.8 \mathrm{~cm}, \text { left } \\
\text { inguinal gonad } 0.6 \times 0.6 \mathrm{~cm}\end{array}$ \\
$\begin{array}{l}1.8 \mathrm{~cm} \text { phallic structure with ventral urethra, fused labioscro- } \\
\text { tal structure, hyperpigmented with ruggae. No introitus or } \\
\text { vaginal opening. No palpable gonads }\end{array}$ & $\begin{array}{l}\text { No uterus: right pelvic gonad } 0.65 \times 0.68 \times 0.7 \mathrm{~cm} ; \text { left } \\
\text { inguinal gonad } 0.9 \times 0.12 \times 0.6 \mathrm{~cm}\end{array}$ \\
\hline
\end{tabular}

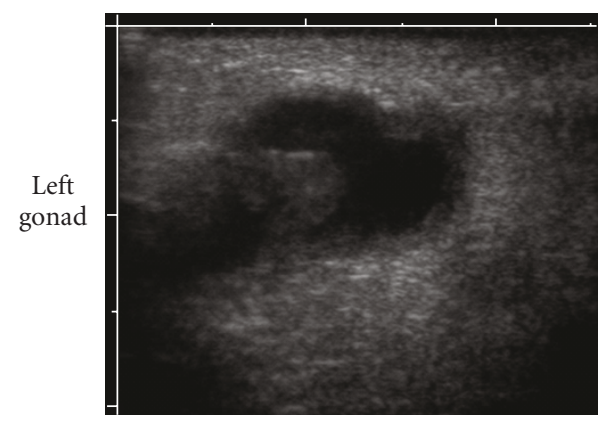

(a)

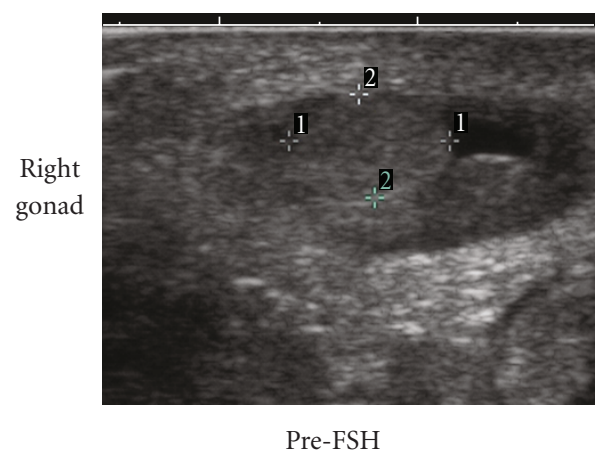

(c)

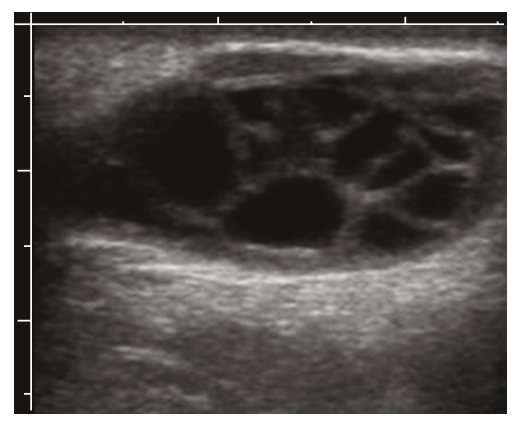

(b)

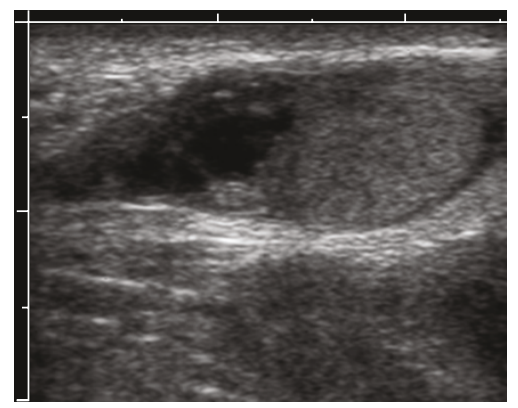

Post-FSH

(d)

FIGURE 1: Case 1: pelvic ultrasound pre- and post-FSH; left gonad pre-FSH: $0.6 \mathrm{~cm} \times 0.4 \mathrm{~cm} \times 0.4 \mathrm{~cm}=0.05 \mathrm{cc}$; left gonad post-FSH: $0.8 \mathrm{~cm}$ $\times 0.5 \mathrm{~cm} \times 0.6 \mathrm{~cm}=0.1 \mathrm{cc}$; right gonad pre-FSH: $2.4 \mathrm{~cm} \times 1.3 \mathrm{~cm} \times 1.6 \mathrm{~cm}=2.61 \mathrm{cc}$; right gonad post-FSH: $1.9 \mathrm{~cm} \times 0.9 \mathrm{~cm} \times 1.2 \mathrm{~cm} \times$ $1.07 \mathrm{cc}$ (normal ovarian volume for age [3]: $1.05 \mathrm{cc}$, SD 0.67, normal testicular volume [4] $\leq 1 \mathrm{cc}$ ).

\begin{abstract}
2.2. Methods. Each infant was given subcutaneous recombinant human FSH (rhFSH-alpha, Gonal-f RFF manufactured by Merck Serono SA) at 25 units per day for 3 days. The dose was extrapolated from adult literature as per the treatment guidelines for in vitro fertilization. The adult dose of rhFSH is $150 \mathrm{IU}$ subcutaneous daily. When this dose is adjusted using body surface area $\left(150 \mathrm{IU} / 1.73 \mathrm{~m}^{2}\right)$, the equivalent dose for a newborn approximates $25 \mathrm{IU}$. Ultrasound evaluation was performed after FSH was administered (day 4) and compared to ultrasounds obtained previously (range: 4 days to 3 months prior). HCG stimulation tests were performed by administering Pregnyl 500 units $/ 1.73 \mathrm{~m}^{2}$ intramuscularly once, with testosterone concentration drawn 72 hours later. Testosterone and estradiol were measured by HPLC with a detection limit of $3 \mathrm{ng} / \mathrm{dl}$ and $0.1 \mathrm{ng} / \mathrm{dl}$, respectively (Esoterix).
\end{abstract}

Case 1. Pelvic ultrasound performed when the infant was 2 months old demonstrated a right inguinal gonad, considered to be consistent with testicular tissue. The left inguinal canal had minimal gonadal tissue with surrounding fluid. No uterus was identified, and no evidence of a tumor was found. Serum testosterone at 3 days of life was $0.5 \mathrm{nmol} / \mathrm{L}(15 \mathrm{ng} / \mathrm{dl})$. Two months later, it had increased to $3.4 \mathrm{nmol} / \mathrm{L}(97 \mathrm{ng} / \mathrm{dl})$ and prior to 4 months was $7.6 \mathrm{nmol} / \mathrm{L}(220 \mathrm{ng} / \mathrm{dl})$.

A recombinant FSH stimulation test was performed when the infant was 4 months old. The post-FSH ultrasound revealed numerous gonadal cystic structures, with more on the left gonad than on the right, suggesting these gonads represented ovotestes (Figure 1). Once again, no uterus was seen. Post-FSH estradiol was $0.2 \mathrm{nmol} / \mathrm{L}(5.6 \mathrm{ng} / \mathrm{dl})$. Serum testosterone at that time was $12.5 \mathrm{nmol} / \mathrm{L}(360 \mathrm{ng} / \mathrm{dl})$. The elevated testosterone concentration likely was not a result of 


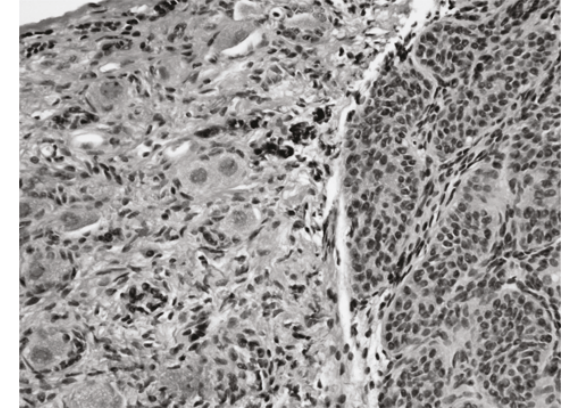

(a)

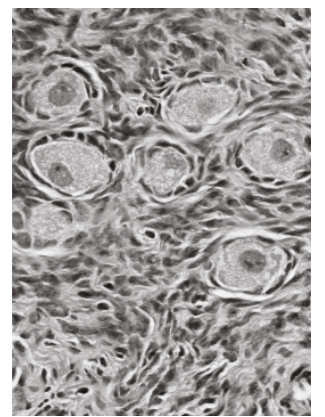

(b)

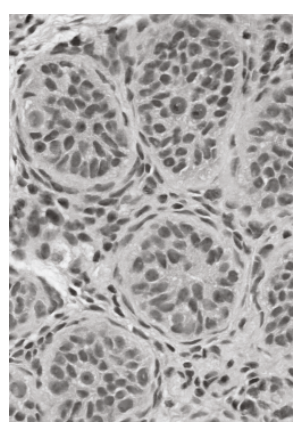

(c)

FIgURE 2: Histopathologic features from the bilateral gonadal excisions (representative images from Case 3) demonstrated features of ovotestes (a)-(c) with ovarian stroma and primary and primordial follicles (b) in close proximity to seminiferous tubules with testicular stroma (c). Cases 1 and 2 demonstrated similar histopathologic findings, with the gonadal biopsies submitted for microscopic examination. (hematoxylin and eosin staining, original magnification: (a) 200x, (b) and (c) 400x).

FSH stimulation but represented physiologic mini-puberty of infancy [5]; however, we have no control data for confirmation.

On laparoscopy, Mullerian remnants were noticed consisting of a gonad and fallopian tube adjacent to the gonad with a fimbriated end noted bilaterally. On the right, mullerian structures were observed consistent with a fallopian tube. The infant underwent bilateral oophorectomy, right orchidopexy, and bilateral inguinal hernia repair. Histopathologic examination of the right and left gonadal biopsies revealed features of an ovotestis with both ovarian and testicular tissue present (Figures 2(a)-2(c)). The ovarian tissue was comprised of ovarian stroma and primordial and primary follicles (Figures 2(a) and 2(b)) . The testicular tissue demonstrated seminiferous tubules with occasional spermatogonia and testicular stroma (Figures 2(a) and $2(c))$. The histopatho logic features of the ovotestis tissue from all three cases were similar, as illustrated in Figure 2 (representative images from Case 3 ). Case 3 is representative of histopathology of the ovotestes seen in all 3 cases. There is no difference in the ovotestes histopathology among the 3 cases).

Microscopic examination of the entirely submitted umbilical and right inguinal hernia sac tissues demonstrated features associated only with typical hernia sacs. Chromosome studies from gonadal biopsy tissues revealed 46, XX and were negative for SRY.

Case 2. Pelvic ultrasound performed when the infant was 2 weeks old demonstrated a small uterus with an endometrial stripe and absent ovaries. Bilateral inguinal hernias with apparent gonads in the hernia sacs also were seen (Figure 3). No cysts were detected within the gonads. Associated epididymal tissue appeared to be present, suggesting that the gonads likely represented testicles. Serum testosterone concentration was $6.9 \mathrm{nmol} / \mathrm{L}(198 \mathrm{ng} / \mathrm{dl})$ when the child was 16 days old.

An HCG stimulation test was administered when the child was 6 weeks old. Post-HCG testosterone concentration was $4.5 \mathrm{nmol} / \mathrm{L}$ (131 ng/dl). A recombinant FSH stimulation test was performed. The post-FSH ultrasound demonstrated a new single cyst in the left gonad (Figure 3). The cyst had the appearance of a follicle, suggesting that this gonad likely represented an ovary. Post-FSH serum testosterone was $6.6 \mathrm{nmol} / \mathrm{L}$ (190 ng/dl), and estradiol $0.02 \mathrm{nmol} / \mathrm{L}$ $(0.5 \mathrm{ng} / \mathrm{dl})$. The estradiol was this low possibly because the ovarian tissue may have been dysfunctional and resistant but not anatomically dysgenetic.

On laparoscopy, a right intraabdominal gonad was observed with mullerian remnant and no vas deferens and on the left an inguinal gonad was observed with a mullerian remnant. The infant underwent bilateral oophorectomy, orchidopexy, and inguinal hernia repair. Upon histopathologic examination, the bilateral gonadal biopsies revealed both ovarian and testicular tissue similar to that illustrated in Figure 2 (representative images from Case 3), confirming the presence of an ovotestis. In addition, a fallopian tube with epididymal tissue in close proximity was identified. The completely submitted left hernia sac showed only features of a typical hernia sac. Chromosome studies from gonadal biopsy tissues revealed $46, \mathrm{XX}$.

Case 3. Pelvic ultrasound performed when the infant was 2 weeks old demonstrated a rounded, relatively homogeneous structure in the right hemipelvis consistent with a gonad. Because no follicles were seen, it was difficult to determine if this structure represented an ovary or a testicle. A second gonadal structure with a central echogenic stripe (typical of the mediastinum of a testicle) was seen in the left hemipelvis. The apparent mediastinum suggested this gonad represented a testicle. Surrounding soft tissue material suggested an epididymis, further suggesting the gonad was a testicle. An HCG stimulation test performed when the infant was 2 weeks old resulted in a serum testosterone increase from $0.9 \mathrm{nmol} / \mathrm{L}(27 \mathrm{ng} / \mathrm{dl})$ to $9.5 \mathrm{nmol} / \mathrm{L}$ (273 ng/dl).

A recombinant FSH stimulation test was performed when the child was 3 weeks old. The post-FSH ultrasound was unchanged (Figure 4). Post-FSH, serum testosterone was $7.3 \mathrm{nmol} / \mathrm{L}(210 \mathrm{ng} / \mathrm{dl})$, and estradiol $<0.02 \mathrm{nmol} / \mathrm{L}$ $(<0.5 \mathrm{ng} / \mathrm{dl})$, see Table 2 . 


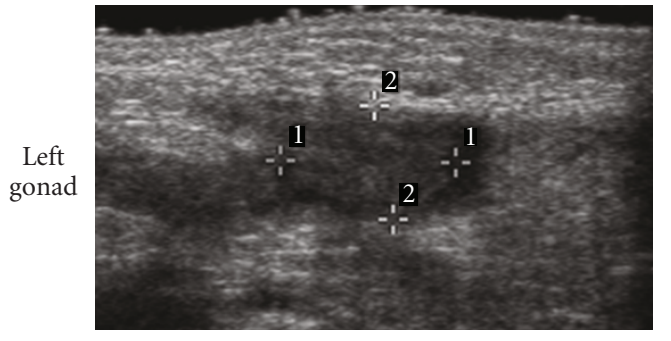

(a)

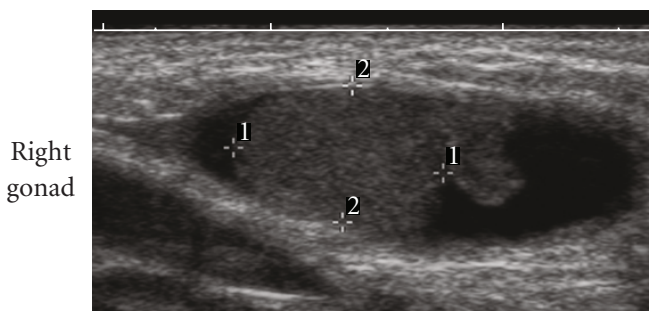

Pre-FSH

(c)

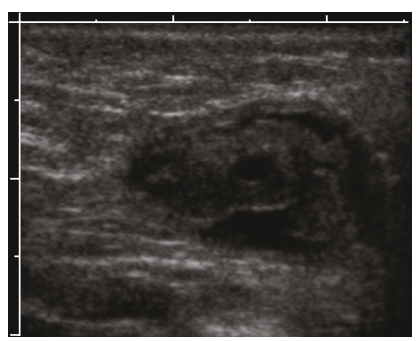

(b)

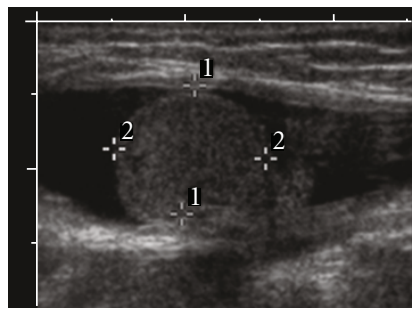

Post-FSH

(d)

Figure 3: Case 2: Pelvic ultrasound pre- and post-FSH. Left gonad pre-FSH: $1.0 \mathrm{~cm} \times 0.6 \mathrm{~cm} \times 0.6 \mathrm{~cm}=0.19 \mathrm{cc}$. Left gonad post-FSH: $1.8 \mathrm{~cm} \times 1.1$ (3rd dimension not measured). Right gonad pre-FSH: $0.9 \mathrm{~cm} \times 0.6 \mathrm{~cm} \times 0.8 \mathrm{~cm}=0.23 \mathrm{cc}$. Right gonad post-FSH: $0.9 \mathrm{~cm} \times$ $1.3 \mathrm{~cm} \times 0.9 \mathrm{~cm}=0.55 \mathrm{cc}$ (normal ovarian volume for age $=1.06 \mathrm{cc}$, SD 0.96 , normal testicular volume $\leq 1 \mathrm{cc})$.

TABLE 2: Steroids pre- and post-FSH including normative data.

\begin{tabular}{|c|c|c|c|c|c|c|c|}
\hline \multirow{2}{*}{$\begin{array}{l}\text { Case } \\
1\end{array}$} & \multirow{2}{*}{$\begin{array}{c}\text { Estradiol pre FSH } \\
(\mathrm{nmol} / \mathrm{L})\end{array}$} & \multicolumn{2}{|c|}{$\begin{array}{l}\text { Estradiol post-FSH } \\
(\mathrm{nmol} / \mathrm{L})\end{array}$} & \multicolumn{2}{|c|}{$\begin{array}{c}\text { Testosterone pre-FSH } \\
(\mathrm{nmol} / \mathrm{L})\end{array}$} & \multicolumn{2}{|c|}{$\begin{array}{c}\text { Testosterone post-FSH } \\
(\mathrm{nmol} / \mathrm{L})\end{array}$} \\
\hline & & 0.2 & Nl: 2-4 & 7.6 & Nl: 2-4 & 12.5 & $\mathrm{Nl}: 2-4$ \\
\hline 2 & Not tested & 0.02 & Nl: 6-9 & 4.5 & Nl: 6-9 & 6.6 & Nl: 6-9 \\
\hline 3 & Not tested & $<0.02$ & NL: 3-6 & 6 & NL: 3-6 & 7.3 & NL: 3-6 \\
\hline
\end{tabular}

Laparoscopy revealed Mullerian remnants bilaterally. The infant underwent bilateral oophorectomy, orchidopexy, and inguinal hernia repair. Histopathologic examination of the bilateral gonadal biopsies and excisions demonstrated features of ovotestes, as illustrated in Figure 2 (representative images from Case 3). The ovarian tissue contained typical stroma with relatively frequent primary and primordial follicles (Figure 2(b)). Microscopic examination of the entirely submitted bilateral inguinal hernia sac tissues failed to reveal fallopian, epididymal, uterine, or Mullerian structures. Chromosome studies with FISH analysis from the biopsy samples revealed low level mosaicism for XXY in 1 percent of cultured cells analyzed; the rest were 46XX.

\section{Discussion}

In North America, ovotesticular DSD is a rare occurrence. The highest incidence is found in Africa [6].

Human menopausal gonadotropins (hMG) or ultrasonography has been used in the past to diagnose ovotesticular DSD, but both tests have limitations. Mendez et al. [7] demonstrated that an hMG challenge was able to identify ovarian tissue based on a specified rise in estradiol; however, the hMG challenge required waiting 6 to 8 weeks after a 4-day hCG stimulation test was performed and monitoring estradiol concentrations for up to 14 days. Sonographic evaluation of ovotestes also has been reported, but not all cases of ovotestes are detected with ultrasoundl [8].

Because of the duration of time needed with the hMG challenge and the inadequate sensitivity of ultrasonography, our Gender Medicine Team used an innovative approach, FSH stimulation testing, in three cases (two Hispanic infants and one African American/Hispanic infant) in an attempt to overcome these limitations. Using adult studies, a safe dose was extrapolated, adjusting for body surface area.

The FSH method was applied as an innovative tool to increase the sensitivity of ultrasound in the evaluation of ovotestes and to determine the presence of ovarian tissue. The use of rhFSH in these cases was intended as a diagnostic tool and was considered a nonexperimental innovation, in which the outcome of an innovation can be reliably predicted $[9,10]$. Non-experimental innovation is a common practice in pediatrics, and consent to use it should be obtained from the patient or the patient's surrogate $[10,11]$. 


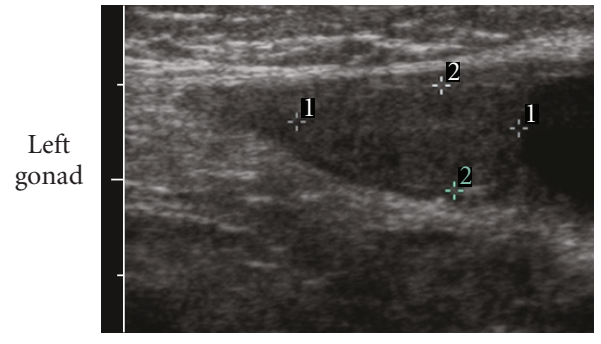

(a)

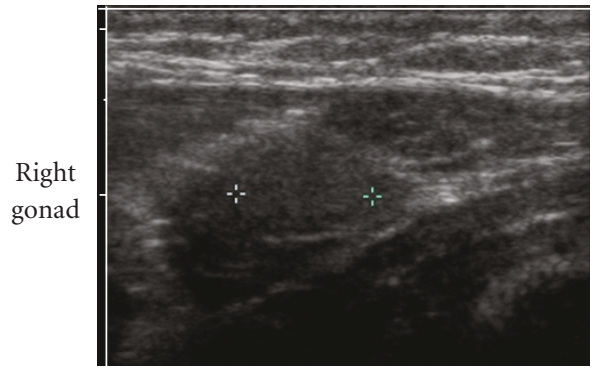

Pre-FSH

(c)

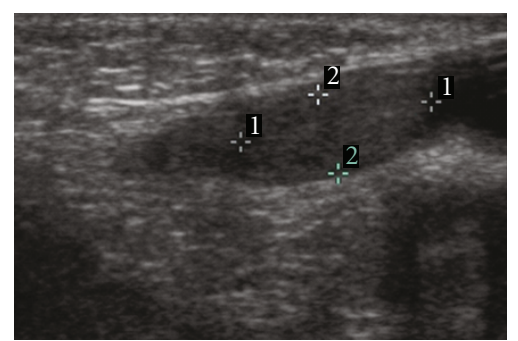

(b)

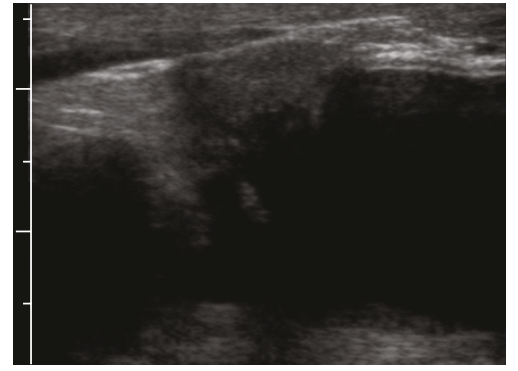

Post-FSH

(d)

Figure 4: Case 3: Pelvic ultrasound pre- and post-FSH. Left gonad pre-FSH: $1.2 \mathrm{~cm} \times 0.6 \mathrm{~cm} \times 0.8 \mathrm{~cm}=0.3 \mathrm{cc}$. Left gonad post-FSH: $1.3 \mathrm{~cm}$ $\times 0.5$ (third dimension not measured). Right gonad pre-FSH: $0.7 \mathrm{~cm} \times 0.7 \mathrm{~cm} \times 0.5 \mathrm{~cm}=0.13 \mathrm{cc}$. Right gonad post-FSH: $0.8 \mathrm{~cm} \times 0.5 \mathrm{~cm}$ $\times 0.8 \mathrm{~cm}=0.17 \mathrm{cc}$ (normal ovarian volume for age $=1.06 \mathrm{cc}$, SD 0.96 , normal testicular volume $\leq 1 \mathrm{cc})$

The presence of follicles in portions of the gonadal tissue after FSH stimulation suggested the diagnosis of ovotestis by ultrasound in two of the three infants. All three cases later were confirmed by gonadal biopsy. In one case, follicles in the gonads developed only after FSH stimulation. In a second case, follicles increased in size and number after FSH stimulation, providing a more conclusive sonographic diagnosis of ovotestes. In the third case, no follicles were seen before or after FSH stimulation. No abdominal findings were manifested, and there was no pathologic evidence of the ovaries when biopsy results were available. In retrospect, it may have been helpful to have pre-FSH estradiol concentrations. It appears that a longer duration of FSH therapy, or delayed estradiol follow-up, is required to appreciate an increase in estradiol. No side effects were reported or observed, including breast development, clitoromegaly, vaginal bleeding, vomiting, diarrhea or fluid retention.

The FSH stimulation test, when compared with the previously reported hMG challenge, was of shorter duration and utilized imaging of stimulated gonadal tissue in place of estradiol concentrations. In each of our cases, the FSH stimulation testing provided for earlier diagnosis and earlier visualization of the gonads, thereby allowing the parents to consider the therapeutic options at an earlier date than otherwise was possible [12]. Identifying ovotestes facilitates the discussion of sex assignment at an earlier date than otherwise possible. When these cases demonstrated that FSH stimulation could be a useful diagnostic tool, we submitted a proposal to our IRB to test the sensitivity and specificity of this hypothesis, which was recently approved. There are no previous reports of FSH being used for this specific indication.

A recent report from Steinmetz et al. examined the use of combined LH and FSH injections to evaluate testosterone, estrogen, and inhibin A response in a cohort of patients with DSD [13]. The study cohort included patients with DSD (mean age: 8.4 years) and females with congenital adrenal hyperplasia $(\mathrm{CAH})$ as the positive-ovarian tissue controls [13]. The subjects received the adult recommended dose of LH/FSH, 150 IU, subcutaneously daily for a total of 3 days [13]. No imaging studies were obtained after the stimulation was performed. A robust estradiol and inhibin A response occurred in the DSD group, similar to the one observed in the $\mathrm{CAH}$ females, demonstrating the effectiveness of combined LH/FSH injections to identify functional ovarian tissue by measuring in blood samples specific secretory products of ovarian tissue [13].

In contrast to that study, our approach aimed to increase the yield of ultrasonography by stimulating follicle formation from ovarian tissue in infants with DSD. Hence, we opted to use $\mathrm{FSH}$, the gonadotropin with a predominant stimulatory role in follicle formation. The approach of measuring preand poststimulation concentrations of estradiol and inhibin A has the advantage of providing biochemical identification of ovarian tissue, and, in combination with the ultrasanography, should provide an ideal substitute for laparoscopy, which is considered the gold standard. Our approach with FSH stimulation relies on the principle of inducing follicle formation to enhance the yield of ultrasonography and, therefore, does not require a significant amount of blood to be drawn. As a safety precaution, we adjusted the FSH 
dose for body surface area, which significantly decreased the concentration of the FSH delivered to our infant subjects and may have limited the formation of follicles in our third case. This infant was the youngest patient, and the FSH stimulation test was performed when the infant was 2 weeks old. We speculate that a higher dose of FSH may be necessary in neonates as they undergo a physiological "mini puberty" period in which LH and FSH are known to be the highest in the life cycle [5].

\section{Conclusion}

Using recombinant FSH to stimulate the development of sonographically identifiable follicles in ovotestes allowed us to establish the diagnosis of ovotesticular DSD earlier in two of our three cases. In conclusion, the use of FSH stimulation followed by ultrasonography and the measurements of estradiol and inhibin A may help preclude the need for laparoscopy and biopsy in this population and thereby contribute to the attempt to provide less invasive interventions in the treatment of ambiguous genitalia. Nonetheless, as more studies are needed in this area, laparoscopy remains the gold standard. The confirmation of our hypothesis of the advantage of combining FSH stimulation and ultrasonography will require further studies to establish sensitivity and specificity. Furthermore, additional research is required to address the optimal recombinant FSH dosing, the timing of followup evaluation, and the addition of hormonal assays to confirm the early diagnosis of ovotesticular DSD. We, therefore, are continuing our studies under the recently approved IRB, and our study is open for recruitment.

\section{Acknowledgments}

We thank Dr. Amaral for contributing to the diagnostic work up of the third case and Dr. Andrea Balazs for contributing to the hospital care of the second case.

\section{References}

[1] I. A. Hughes, C. Houk, S. F. Ahmed, and P. A. Lee, "Consensus statement on management of intersex disorders," Archives of Disease in Childhood, vol. 91, no. 7, pp. 554-563, 2006.

[2] M. Filicori, G. E. Cognigni, C. Tabarelli, et al., "Stimulation and growth of antral ovarian follicles by selective LH activity administration in women," Journal of Clinical Endocrinology and Metabolism, vol. 87, no. 3, pp. 1156-1161, 2002.

[3] H. L. Cohen, M. A. Shapiro, F. S. Mandel, and M. L. Shapiro, "Normal ovaries in neonates and infants: a sonographic study of 77 patients 1 day to 24 months old," American Journal of Roentgenology, vol. 160, no. 3, pp. 583-586, 1993.

[4] F. G. Cassorla, S. M. Golden, R. D. Johnsonbaugh, et al., "Testicular volume during early infancy," Journal of Pediatrics, vol. 99, no. 5, pp. 742-743, 1981.

[5] M. M. Grumbach, "Commentary: a window of opportunity: the diagnosis of gonadotropin deficiency in the male infant," Journal of Clinical Endocrinology and Metabolism, vol. 90, no. 5, pp. 3122-3127, 2005.

[6] G. Krob, A. Braun, and U. Kuhnle, "True hermaphroditism: geographical distribution, clinical findings, chromosomes and gonadal histology," European Journal of Pediatrics, vol. 153, no. 1, pp. 2-10, 1994.

[7] J. P. Mendez, R. Schiavon, L. Diaz-Cueto, et al., "A reliable endocrine test with human menopausal gonadotropins for diagnosis of true hermaphroditism in early infancy," Journal of Clinical Endocrinology and Metabolism, vol. 83, no. 10, pp. 3523-3526, 1998.

[8] W. Eberenz, H. K. Rosenberg, T. Moshang, J. Chatten, and M. A. Keating, "True hermaphroditism: sonographic demonstration of ovotestes," Radiology, vol. 179, no. 2, pp. 429-431, 1991.

[9] A. M. Reitsma and J. D. Moreno, Eds., Ethical Guidelines for Innovative Surgery, University Publishing, Hagerstown, Md, USA, 2006.

[10] J. W. Jones, L. B. McCullough, and B. W. Richman, "Ethics of surgical innovation to treat rare diseases," Journal of Vascular Surgery, vol. 39, no. 4, pp. 918-919, 2004.

[11] M. F. McKneally and A. S. Daar, "Introducing new technologies: protecting subjects of surgical innovation and research," World Journal of Surgery, vol. 27, no. 8, pp. 930-934, 2003.

[12] S. L. French, J. Amaral, L. M. Rodriguez, S. K. Gunn, A. E. Schlesinger, and L. P. Karaviti, "Is laparoscopy necessary for evaluation of hermaphroditism?" in Proceedings of the 87th Annual Endocrine Society Meeting (ENDO '05), San Diego, Calif, USA, June 2005.

[13] L. Steinmetz, M. N. Rocha, C. A. Longui, et al., "Inhibin A production after gonadotropin stimulus: a new method to detect ovarian tissue in ovotesticular disorder of sex development," Hormone Research, vol. 71, no. 2, pp. 94-99, 2009. 